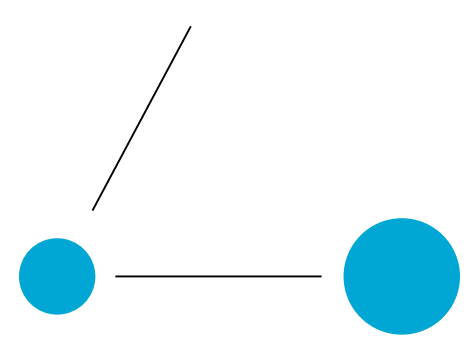

A N N A L E S HENRI LEBESGUE

\title{
LAURENT THOMANN
}

\section{GROWTH OF SOBOLEV NORMS FOR LINEAR SCHRÖDINGER OPERATORS}

\section{CROISSANCE DE NORMES SOBOLEV POUR DES OPERATEURS SCHRÖDINGER L INÉA IRES}

ABstract. - We give an example of a linear, time-dependent, Schrödinger operator with optimal growth of Sobolev norms. The construction is explicit, and relies on a comprehensive study of the linear Lowest Landau Level equation with a time-dependent potential.

RÉSumÉ. - Nous donnons un exemple d'un opérateur de Schrödinger linéaire, dépendant du temps, avec une croissance optimale des normes de Sobolev. La construction est explicite, et s'appuie sur une étude complète de l'équation linéaire de plus bas niveau de Landau avec un potentiel dépendant du temps.

Keywords: Linear Schrödinger equation, time-dependent potential, growth of Sobolev norms, reducibility.

2020 Mathematics Subject Classification: 35Q41, 35B08.

DOI: https://doi.org/10.5802/ahl.111

(*) The author is supported by the grants "BEKAM" ANR-15-CE40-0001 and "ISDEEC" ANR16-CE40-0013. 


\section{Introduction and main result}

The aim of this paper is to present an example of linear, time-dependent, Schrödinger operator which exhibits optimal polynomial growth of Sobolev norms. Moreover, this operator takes the form $\widetilde{H}+\mathscr{L}(t)$, where $\widetilde{H}$ is an elliptic operator with compact resolvent and where the perturbation $\mathscr{L}(t)$ is a small, time-dependent, bounded self-adjoint operator. Our construction is actually entirely explicit and it is based on the study of linear Lowest Landau Level equations (LLL) with a time-dependent potential.

In Maspero-Robert [MR17], the authors study linear Schrödinger operators, obtain global well-posedness results and prove very precise polynomial bounds on the possible growth of Sobolev norms under general conditions (see Assumption 1.1 below). We show here that these bounds are optimal.

Our setting is the following: consider the 2-dimensional harmonic oscillator

$$
H=-\left(\partial_{x}^{2}+\partial_{y}^{2}\right)+\left(x^{2}+y^{2}\right)=-4 \partial_{z} \partial_{\bar{z}}+|z|^{2},
$$

where $z=x+i y, \partial_{z}=\frac{1}{2}\left(\partial_{x}-i \partial_{y}\right)$. This operator acts on the space

$$
\widetilde{\mathcal{E}}=\left\{u(z)=e^{-\frac{|z|^{2}}{2}} f(z), f \text { entire holomorphic }\right\} \cap \mathscr{S}^{\prime}(\mathbb{C}),
$$

and if we define the Bargmann-Fock space $\mathcal{E}$ by

$$
\mathcal{E}=\left\{u(z)=e^{-\frac{|z|^{2}}{2}} f(z), f \text { entire holomorphic }\right\} \cap L^{2}(\mathbb{C}),
$$

then the so-called special Hermite functions $\left(\varphi_{n}\right)_{n \geqslant 0}$ given by

$$
\varphi_{n}(z)=\frac{z^{n}}{\sqrt{\pi n !}} e^{-\frac{|z|^{2}}{2}}
$$

form a Hilbertian basis of $\mathcal{E}$, and are eigenfunctions of $H$, namely

$$
H \varphi_{n}=2(n+1) \varphi_{n}, \quad n \geqslant 0 .
$$

Let $0 \leqslant \tau<1$ and set $\rho(\tau)=\frac{1}{2(1-\tau)} \in[1 / 2, \infty)$. We define the operator $\widetilde{H}_{\tau}=$ $(H+1)^{\rho(\tau)}$, which in turn defines the scale of Hilbert spaces $\left(\widetilde{\mathbb{H}}_{\tau}^{s}\right)_{s \geqslant 0}$ by

$$
\widetilde{\mathbb{H}}_{\tau}^{s}=\left\{u \in L^{2}(\mathbb{C}), \widetilde{H}_{\tau}^{s / 2} u \in L^{2}(\mathbb{C})\right\} \cap \mathcal{E}, \quad \widetilde{\mathbb{H}}_{\tau}^{0}=\mathcal{E},
$$

and we denote by $L$ the Lebesgue measure on $\mathbb{C}$.

For $X$ a Banach space, we denote by $\mathcal{C}_{b}(\mathbb{R} ; X)$ the subspace of $\mathcal{C}(\mathbb{R} ; X)$ composed of bounded functions:

$$
\mathcal{C}_{b}(\mathbb{R} ; X)=\left\{t \mapsto u(t) \in \mathcal{C}(\mathbb{R} ; X): \sup _{t \in \mathbb{R}}\|u(t)\|_{X}<+\infty\right\}
$$

Similarly, for all $k \in \mathbb{N}$ we define the space $\mathcal{C}_{b}^{k}(\mathbb{R}, X)$ by

$$
\mathcal{C}_{b}^{k}(\mathbb{R} ; X)=\left\{u \in \mathcal{C}_{b}(\mathbb{R} ; X): \partial_{t}^{j} u \in \mathcal{C}_{b}(\mathbb{R} ; X), \forall 0 \leqslant j \leqslant k\right\} .
$$

Let $0 \leqslant \tau<1$ and $s \geqslant 0$. For a family $(\mathscr{L}(t))_{t \in \mathbb{R}}$ of continuous linear mappings

$$
\mathscr{L}(t): \widetilde{\mathbb{H}}_{\tau}^{s} \longrightarrow \widetilde{\mathbb{H}}_{\tau}^{s}
$$

we consider the following assumptions : 
Assumption 1.1. - $(\mathscr{L}(t))_{t \in \mathbb{R}}$ is a family of linear operators which satisfies:

(i) One has $t \longmapsto \mathscr{L}(t) \in \mathcal{C}_{b}\left(\mathbb{R} ; \mathcal{L}\left(\widetilde{\mathbb{H}}_{\tau}^{s}\right)\right)$ for all $s \geqslant 0$.

(ii) For every $t \in \mathbb{R}, \mathscr{L}(t)$ is symmetric w.r.t. the scalar product of $\widetilde{\mathbb{H}}_{\tau}^{0}$,

$$
\int_{\mathbb{C}} \bar{v} \mathscr{L}(t) u d L=\int_{\mathbb{C}} u \overline{\mathscr{L}(t) v} d L, \quad \forall u, v \in \widetilde{\mathbb{H}}_{\tau}^{0} .
$$

(iii) The family $(\mathscr{L}(t))_{t \in \mathbb{R}}$ is $\widetilde{H}_{\tau}^{\tau}$-bounded in the sense that $t \longmapsto\left[\mathscr{L}(t), \widetilde{H}_{\tau}\right] \widetilde{H}_{\tau}^{-\tau} \in$ $\mathcal{C}_{b}\left(\mathbb{R}, \mathcal{L}\left(\widetilde{\mathbb{H}}_{\tau}^{s}\right)\right)$ for all $s \geqslant 0$.

(iv) For all $\ell \in \mathbb{N}$, one has $t \longmapsto \mathscr{L}(t) \in \mathcal{C}_{b}^{\ell}\left(\mathbb{R} ; \mathcal{L}\left(\widetilde{\mathbb{H}}_{\tau}^{s} ; \widetilde{\mathbb{H}}_{\tau}^{s-\ell \tau}\right)\right)$ for all $s \geqslant 0$.

Finally, for $s \geqslant 0$, we consider the initial value problem

$$
\left\{\begin{array}{l}
i \partial_{t} u=\left(\widetilde{H}_{\tau}+\mathscr{L}(t)\right) u, \quad(t, z) \in \mathbb{R} \times \mathbb{C}, \\
u(t, \cdot)_{\mid t=t_{0}}=u_{0} \in \widetilde{\mathbb{H}}_{\tau}^{s},
\end{array}\right.
$$

and we are able to state our main result:

Theorem 1.2. - For any $0 \leqslant \tau<1$ and any $\epsilon>0$, there exists a family of linear operators $(\mathscr{L}(t))_{t \in \mathbb{R}}$ which satisfies Assumption 1.1, so that for all $s \geqslant 0$ :

(i) There exists $C_{s}>0$ such that

$$
\sup _{t \in \mathbb{R}}\|\mathscr{L}(t)\|_{\mathcal{L}\left(\widetilde{\mathbb{H}}_{\tau}^{s}\right)} \leqslant C_{s} \epsilon .
$$

(ii) The problem (1.1) is globally well-posed in $\widetilde{\mathbb{H}}_{\tau}^{s}$ : for any $u_{0} \in \widetilde{\mathbb{H}}_{\tau}^{s}$, there exists a unique solution $u(t):=\mathcal{U}\left(t, t_{0}\right) u_{0}$ such that $u \in \mathcal{C}\left(\mathbb{R}, \widetilde{\mathbb{H}}_{\tau}^{s}\right)$ to (1.1). Moreover, $\mathcal{U}$ has the group property

$$
\mathcal{U}\left(t_{2}, t_{1}\right) \mathcal{U}\left(t_{1}, t_{0}\right)=\mathcal{U}\left(t_{2}, t_{0}\right), \quad \mathcal{U}(t, t)=I_{d}, \quad \forall t, t_{1}, t_{2} \in \mathbb{R},
$$

and $\mathcal{U}$ is unitary in $\widetilde{\mathbb{H}}_{\tau}^{0}$

$$
\left\|\mathcal{U}\left(t, t_{0}\right) u_{0}\right\|_{\widetilde{\mathbb{H}}_{\tau}^{0}}=\left\|u_{0}\right\|_{\widetilde{\mathbb{H}}_{\tau}^{0}}, \quad \forall t \in \mathbb{R} .
$$

(iii) Any solution to (1.1), with initial condition $u_{0} \in \widetilde{\mathbb{H}}_{\tau}^{s}$, satisfies for all $t \in \mathbb{R}$

$$
\left\|\mathcal{U}\left(t, t_{0}\right) u_{0}\right\|_{\widetilde{\mathbb{H}}_{\tau}^{s}} \leqslant C\left\|u_{0}\right\|_{\widetilde{\mathbb{H}}_{\tau}^{s}}\left\langle\epsilon\left(t-t_{0}\right)\right\rangle^{\frac{s}{2(1-\tau)}} .
$$

(iv) There exists a nontrivial initial condition $u_{0} \in \cap_{k \geqslant 1} \widetilde{\mathbb{H}}_{\tau}^{k}$ such that the corresponding solution to (1.1) satisfies for all $t \in \mathbb{R}$

$$
\left\|\mathcal{U}\left(t, t_{0}\right) u_{0}\right\|_{\widetilde{\mathbb{H}}_{\tau}^{s}} \geqslant c\left\|u_{0}\right\|_{\widetilde{\mathbb{H}}_{\tau}^{s}}\left\langle\epsilon\left(t-t_{0}\right)\right\rangle^{\frac{s}{2(1-\tau)}} .
$$

Actually, items (ii) and (iii) directly follow from [MR17, Theorem 1.5]. The novelty in our work is item (iv) which shows that the upper bounds obtained in [MR17, Theorem 1.5] are optimal without further assumptions, even for small perturbations $\mathscr{L}(t)$, see item (i).

It seems that the example of Theorem 1.2 is the first one which covers all the possible values of $0 \leqslant \tau<1$, and it is noticeable the result of Theorem 1.2 is obtained for any value of $0 \leqslant \tau<1$, by essentially the same example, written in different scales of Hilbert spaces. An example of such growth was given in [Del14] in the case $\tau=0$ 
(see also [Mas19] for an alternative proof), and in [BGMR18, Appendix A] in the case $\tau=1 / 2$, but it seems that the other cases were left open.

We stress that our example is an operator which takes the form $\widetilde{H}_{\tau}+\mathscr{L}(t)$, where $\widetilde{H}_{\tau}$ is a (time independent) elliptic operator with compact resolvent and $\mathscr{L}(t)$ a bounded self-adjoint operator. Moreover, this perturbation is small and satisfies indeed for all $\ell \geqslant 0$

$$
\sup _{t \in \mathbb{R}}\left\|\partial_{t}^{\ell} \mathscr{L}(t)\right\|_{\mathcal{L}\left(\widetilde{\mathbb{H}}_{\tau}^{s} ; \widetilde{\mathbb{H}}_{\tau}^{s-\ell \tau}\right)} \leqslant C_{s, \ell} \epsilon
$$

If one allows unbounded perturbations, it is simpler to obtain growth of Sobolev norms, as it is shown by an elementary example given in Appendix A. In this latter context, growth of Sobolev norms can occur even with time-independent operators.

Observe that the $\varphi_{n}$ are the eigenfunctions of $\widetilde{H}_{\tau}$, namely

$$
\widetilde{H}_{\tau} \varphi_{n}=2^{\rho(\tau)}(n+1)^{\rho(\tau)} \varphi_{n}, \quad n \geqslant 0 .
$$

Hence in our example, we see an exact correspondence between the asymptotics of the eigenvalues of $\widetilde{H}_{\tau}$ and the rate of growth for (1.1). If $\rho>1$ (which corresponds to $\tau>1 / 2)$, the operator $\widetilde{H}_{\tau}$ satisfies a gap condition, but in our example, $\partial_{t}^{\ell} \mathscr{L}(t)$ is not regular enough (see item $(i v)$ in Assumption 1.1) to meet the hypotheses of [MR17, Theorems 1.8 and 1.9], in which better upper bounds are obtained.

Let us recall the following characterization of the Sobolev spaces $\widetilde{\mathbb{H}}_{\tau}^{s}$. By [GGT19, Lemma C.1], for any $s \geqslant 0$, there exist $c, C>0$ such that for all $u \in \widetilde{\mathbb{H}}_{\tau}^{s}$

$$
c\left\|\langle z\rangle^{s \rho(\tau)} u\right\|_{L^{2}(\mathbb{C})} \leqslant\|u\|_{\widetilde{\mathbb{H}}_{\tau}^{s}} \leqslant C\left\|\langle z\rangle^{s \rho(\tau)} u\right\|_{L^{2}(\mathbb{C})}, \quad\langle z\rangle=\left(1+|z|^{2}\right)^{1 / 2} .
$$

As a consequence, in the Bargmann-Fock space, a growth of Sobolev norm corresponds to a transfer of energy in the physical space. In our example, the growth will be induced by a traveling wave. This is in contrast to the previous known examples [BGMR18, Del14, Mas19], where the growth was inherited by a time-periodic phenomenon.

We end this section by reviewing some results on the growth of linear Schrödinger equations on manifolds with time-dependent potentials

$$
i \partial_{t} u+\Delta u+V(t, x) u=0 .
$$

In [Bou99b] Bourgain proves a polynomial bound of the Sobolev norm for (1.2), when $V(t, x)$ is a bounded (real analytic) potential. Moreover, when the potential is quasi-periodic in time he obtains in [Bou99a] a logarithmic bound (see also [Del10, FZ12, GPT13, Wan08a], for more results on norm inflation phenomena in various settings). Delort [Del14] constructs an example with polynomial growth for the harmonic oscillator perturbed by a (time-periodic) pseudo-differential operator of order zero. In [BGMR18], the authors give the example of a time-periodic order one perturbation of the harmonic oscillator which induces polynomial growth. In [ANS19], the authors prove exponential growth of the energy norm for a linear (and nonlinear) harmonic oscillator perturbed by the angular momentum operator (see [ANS19, Theorem 4.5]). We refer to [HM20, Mas19] for more examples with growth of norms and to [BGMR21] for bounds on abstract linear Schrödinger equations. Let us mention the article [LZZ21] in which the authors obtain very precise results on the 
dynamics of a family of perturbations of the harmonic oscillator. Finally, in the recent paper [FR20], Faou and Raphaël give examples of solutions to perturbed harmonic oscillators which grow like $(\log t)^{\alpha}$. Interestingly, although being different to ours, one of their approach relies on the study of the so-called continuous resonant equation (CR) which contains the dynamics of the LLL equation.

\section{The linear LLL equation with time-dependent potential}

We now present our example more in details. Let $W \in L^{\infty}(\mathbb{R} \times \mathbb{C}, \mathbb{R})$ be a realvalued time-dependent potential and consider the linear equation

$$
\left\{\begin{array}{l}
i \partial_{t} u-\delta H u=\Pi(W(t, z) u), \quad(t, z) \in \mathbb{R} \times \mathbb{C}, \quad \delta \in \mathbb{R}, \\
u(t, \cdot)_{\mid t=t_{0}}=u_{0} \in \mathcal{E},
\end{array}\right.
$$

where $\Pi$ is the orthogonal projector on the space $\mathcal{E}$ (the kernel of $\Pi$ is very explicit, see (2.13) below). The equation (2.1) is the linearization of the Lowest Landau Level equation

which is used in the modeling of fast rotating Bose-Einstein condensates. See e.g. the introduction of [GGT19] for physical motivation, and we refer to [ABN06, BBCE17, BBE19, GGT19, Nie07, ST21] for the study of (2.1). Equation (2.1) is a natural mathematical toy model, for which we can try to exhibit some particular dynamics.

The dispersion parameter $\delta \in \mathbb{R}$ does not play a role in the dynamics of equation (2.2). Actually, $u$ solves (2.2) if and only if $v=e^{i \delta t H} u$ solves (2.2) with $\delta=0$. This comes from the crucial property

$$
e^{-i t H} \Pi\left(e^{i t H} a \overline{e^{i t H} b} e^{i t H} c\right)=\Pi(a \bar{b} c), \quad \forall a, b, c \in \mathcal{E},
$$

see [GHT16, Lemma 2.4 and Corollary 2.5]. However, the transformation $v=e^{i \delta t H} u$ does not preserve the left hand side of $(2.1)$, that is why we must keep the parameter $\delta \in \mathbb{R}$ in our study (nonetheless we will see that it does not affect the dynamics of equation (2.1), excepted in the reducibility result stated in Appendix A where we need $\delta \neq 0)$.

In the sequel, by a time translation, we restrict to the case $t_{0}=0$.

In this section, we state global well-posedness results with optimal bounds on the growth of the Sobolev norms for (2.1). We are also able to obtain reducibility results for (2.1), when $W$ is a small quasi-periodic potential, but these results are direct applications of [GT11], thus we have postponed the statements to the Appendix A.

\subsection{Statement of the results}

Our first result concerns the global well-posedness of such an equation under general conditions on $W$. For $s \geqslant 0$, we denote by

$$
L^{2, s}=\left\{u \in L^{2}(\mathbb{C}),\langle z\rangle^{s} u \in L^{2}(\mathbb{C})\right\}, \quad\langle z\rangle=\left(1+|z|^{2}\right)^{1 / 2}
$$


the weighted Lebesgue space and $L_{\mathcal{E}}^{2 s}=L^{2, s} \cap \mathcal{E}$. Then our well-posedness result reads:

Theorem 2.1. - Let $\delta \in \mathbb{R}$ and $W \in L^{\infty}(\mathbb{R} \times \mathbb{C}, \mathbb{R})$. For all $u_{0} \in \mathcal{E}$, there exists a unique solution $u \in \mathcal{C}(\mathbb{R}, \mathcal{E})$ to equation (2.1). Moreover, for every $t \in \mathbb{R}$,

$$
\int_{\mathbb{C}}|u(t, z)|^{2} d L(z)=\int_{\mathbb{C}}\left|u_{0}(z)\right|^{2} d L(z) .
$$

Furthermore, if for some $s>0, u_{0} \in L_{\mathcal{E}}^{2, s}$, then $u(t) \in L_{\mathcal{E}}^{2, s}$ for every $t \in \mathbb{R}$.

A natural question is the control of higher order Sobolev norms of the solution for large times and this will be achieved, under some additional conditions on $W$. The notation $W \in \mathcal{C}_{b_{t}}^{\infty}\left(\mathbb{R} \times \mathbb{R}^{2}, \mathbb{R}\right)$ means that $W$ is continuous and bounded in $t$ and smooth in the variables $(x, y)$. We stress that derivation in the time variable is not needed. For notational convenience, we sometimes identify $(x, y) \in \mathbb{R}^{2}$ and $z=x+i y \in \mathbb{C}$. In particular, for a function of the variables $(x, y)$ we use the notations

$$
\partial_{z}=\frac{1}{2}\left(\partial_{x}-i \partial_{y}\right), \quad \partial_{\bar{z}}=\frac{1}{2}\left(\partial_{x}+i \partial_{y}\right) .
$$

Theorem 2.2. - Let $\delta \in \mathbb{R}$ and $s \geqslant 0$. Assume that $W \in \mathcal{C}_{b_{t}}^{\infty}\left(\mathbb{R} \times \mathbb{R}^{2}, \mathbb{R}\right)$ is such that

$$
\sup _{0 \leqslant k \leqslant\lceil s\rceil}\left\|\partial_{z}^{k} W(t, \cdot)\right\|_{L^{\infty}(\mathbb{C})} \leqslant C_{0}, \quad t \in \mathbb{R}
$$

then any solution to (2.1), with initial condition $u_{0} \in L_{\mathcal{E}}^{2, s}$, satisfies for all $t \in \mathbb{R}$

$$
\left\|\langle z\rangle^{s} u(t)\right\|_{L^{2}(\mathbb{C})} \leqslant C\left\|\langle z\rangle^{s} u_{0}\right\|_{L^{2}(\mathbb{C})}\left\langle C_{0} t\right\rangle^{s},
$$

where the constant $C>0$ only depends on $s \geqslant 0$.

Condition (2.5) is rather natural in the space $\mathcal{E}$. For instance, it is satisfied by the following class of potentials: assume that $V(t, \cdot) \in \mathcal{E}$, uniformly in $t \in \mathbb{R}$, then $W=|V|^{2}$ satisfies (2.5) for all $k \geqslant 0$, by Lemma C.2 and (2.15). We stress that in $(2.5)$ one needs the operator $\partial_{z}\left(\right.$ not $\partial_{x}$ or $\left.\partial_{y}\right)$.

This bound is indeed optimal as shown by the next result:

Theorem 2.3. - Let $\delta \in \mathbb{R}$. For all $\epsilon>0$, there exists $W_{\epsilon} \in \mathscr{S}\left(\mathbb{R} \times \mathbb{R}^{2}, \mathbb{R}\right)$ such that for all $1 \leqslant p \leqslant \infty,\left\|W_{\epsilon}(t, \cdot)\right\|_{L^{p}(\mathbb{C})} \leqslant \epsilon$, and such that for all $k, j \geqslant 0$ and uniformly in time

$$
\left\|\partial_{\bar{z}}^{j} \partial_{z}^{k} W_{\epsilon}(t, \cdot)\right\|_{L^{\infty}(\mathbb{C})} \leqslant \epsilon C_{j k}, \quad t \in \mathbb{R}
$$

and there exists a nontrivial initial condition $u_{0} \in \bigcap_{k \geqslant 0} L_{\mathcal{E}}^{2, k}$ such that the corresponding solution to (2.1) satisfies for all $s \geqslant 0$ and $t \in \mathbb{R}$

$$
\left\|\langle z\rangle^{s} u(t)\right\|_{L^{2}(\mathbb{C})} \geqslant c_{s}\left\|\langle z\rangle^{s} u_{0}\right\|_{L^{2}(\mathbb{C})}\langle\epsilon t\rangle^{s} .
$$

Moreover, we have the following equivalence, when $t \longrightarrow \pm \infty$

$$
\left\|\langle z\rangle^{s} u(t)\right\|_{L^{2}(\mathbb{C})} \sim c^{s} \epsilon^{s}|t|^{s}\left\|u_{0}\right\|_{L^{2}(\mathbb{C})} .
$$


The inequality (2.8) is stated like that in order to give a counterpart to (2.6). Actually, as shown in (2.9), the coefficient of the leading order of the lower term depends on $\left\|u_{0}\right\|_{L^{2}(\mathbb{C})}$ (and not on $\left\|\langle z\rangle^{s} u_{0}\right\|_{L^{2}(\mathbb{C})}$ ). The inequality (2.8) holds true since $u_{0} \in \bigcap_{k \geqslant 0} L_{\mathcal{E}}^{2, k}$ is here fixed, and the constant $c_{s}>0$ depends on $u_{0}$.

Observe that using the notations (2.4), the condition (2.7) can be rephrased as

$$
\left\|\partial_{x}^{j} \partial_{y}^{k} W_{\epsilon}(t, \cdot)\right\|_{L^{\infty}(\mathbb{C})} \leqslant \epsilon C_{j k}, \quad t \in \mathbb{R} .
$$

The result of Theorem 2.3 is a direct consequence of [ST21, Theorem 1.5 and Corollary 1.6] and we can make the explicit choices

$$
u_{0}=\sqrt{\epsilon}\left(\frac{1}{2} \varphi_{0}+i \frac{\sqrt{3}}{2} \varphi_{1}\right), \quad \alpha=\frac{\sqrt{3}}{32 \pi} \epsilon,
$$

and

$$
W_{\epsilon}(t, z)=\frac{\epsilon}{4 \pi}\left|1-i \sqrt{3}\left(e^{-2 i \delta t} z+\alpha t\right)\right|^{2} e^{-\left|e^{-2 i \delta t} z+\alpha t\right|^{2}} .
$$

Actually, in [ST21, Theorem 1.5 and Corollary 1.6] (see also [ST21, equation (2.4)]), unbounded trajectories where constructed for the system

$$
\left\{\begin{array}{l}
i \partial_{t} u-\delta H u=\Pi\left(|v|^{2} u\right), \quad(t, z) \in \mathbb{R} \times \mathbb{C}, \\
i \partial_{t} v-\delta H v=-\Pi\left(|u|^{2} v\right), \\
u(0, \cdot)=u_{0}, v(0, \cdot)=v_{0},
\end{array}\right.
$$

and the idea is here to consider the second equation in (2.11) as given, and to interpret the term $|v|^{2}$ in the first line as a given time-dependent potential.

Notice that the growth of Sobolev norms is not obtained by a periodic potential as in [BGMR18, Del10]. Here, as it is shown in (2.10), the growth is exhibited by a time translation (more precisely, by a magnetic translation in the Bargmann-Fock space).

In general, growth of Sobolev norms is a phenomenon which happens due to resonances of the equation. Recall that the dynamics of the cubic LLL equation $i \partial_{t} u=\Pi\left(|u|^{2} u\right)$ is included in the so-called cubic resonant (CR) equation, which was derived in [FGH16] as a resonant approximation of NLS (we also refer to [GHT16] for a comprehensive study of the (CR) equation).

In the last result of this section we show that if $W$ has additional spacial decay, then the possible growth of the solution of (2.1) enjoys better controls:

Theorem 2.4. - Let $\delta \in \mathbb{R}$ and $s \geqslant 0$ and let $W \in \mathcal{C}_{b_{t}}^{\infty}\left(\mathbb{R} \times \mathbb{R}^{2}, \mathbb{R}\right)$.

(i) Assume that

$$
\sup _{1 \leqslant j \leqslant k \leqslant\lceil s\rceil}\left\|z^{2 k-j} \partial_{z}^{j} W(t, \cdot)\right\|_{L^{\infty}(\mathbb{C})} \leqslant C_{1}, \quad t \in \mathbb{R},
$$

then any solution to (2.1), with initial condition $u_{0} \in L_{\mathcal{E}}^{2, s}$, satisfies for all $t \in \mathbb{R}$

$$
\left\|\langle z\rangle^{s} u(t)\right\|_{L^{2}(\mathbb{C})} \leqslant C\left\|\langle z\rangle^{s} u_{0}\right\|_{L^{2}(\mathbb{C})}\left\langle C_{1} t\right\rangle^{1 / 2}
$$

where the constant $C>0$ only depends on $s \geqslant 0$. 
(ii) Let $\epsilon>0$. Assume that for all $k \geqslant 1$ and uniformly in time

$$
\left\|\langle z\rangle^{2 k} \partial_{z}^{k} W(t, \cdot)\right\|_{L^{\infty}(\mathbb{C})} \leqslant C_{k}, \quad t \in \mathbb{R}
$$

then any solution to (2.1), with initial condition $u_{0} \in L_{\mathcal{E}}^{2, s}$, satisfies for all $t \in \mathbb{R}$

$$
\left\|\langle z\rangle^{s} u(t)\right\|_{L^{2}(\mathbb{C})} \leqslant C\left\|\langle z\rangle^{s} u_{0}\right\|_{L^{2}(\mathbb{C})}\langle t\rangle^{\epsilon}
$$

where the constant $C>0$ depends on $W, s \geqslant 0$ and $\epsilon>0$.

This result is the analogous to [Bou99b, Del10] in which similar bounds are obtained for the linear Schrödinger equation with time-dependent potential, but in our case the proof is much simpler.

The result of Theorem 2.4 shows that growth of Sobolev norms can occur only if $W$ is concentrated in the region $|z| \gg 1$ when $t \longrightarrow \pm \infty$. This is typically the case with the example of the traveling wave exhibited in Theorem 2.3 (see (2.10)).

Under additional conditions on $W$ (analyticity in time and quasi-periodicity) one can show the solutions are indeed bounded, see Theorem B.1.

\subsection{Plan of the paper}

The rest of the paper is organized as follows. We end this section by giving some notations. In Section 3 we study the linear LLL equation (2.1). We are then able to apply these results to prove Theorem 1.2 in Section 4. In Appendix A we give an another example of Schrödinger operator with unbounded orbits and in Appendix B we state a reducibility result for (2.1).

\subsection{Some recalls and notations}

The harmonic oscillator $H$ is defined by

$$
H=-4 \partial_{z} \partial_{z}+|z|^{2}
$$

with the classical notations $z=x+i y$ and

$$
\partial_{z}=\frac{1}{2}\left(\partial_{x}-i \partial_{y}\right), \quad \partial_{\bar{z}}=\frac{1}{2}\left(\partial_{x}+i \partial_{y}\right) .
$$

Recall that the family of the special Hermite functions $\left(\varphi_{n}\right)_{n \geqslant 0}$ is given by

$$
\varphi_{n}(z)=\frac{z^{n}}{\sqrt{\pi n !}} e^{-\frac{|z|^{2}}{2}} .
$$

The family $\left(\varphi_{n}\right)_{n \geqslant 0}$ forms a Hilbertian basis of $\mathcal{E}$ (see [Zhu12, Proposition 2.1]), and the $\varphi_{n}$ are the eigenfunctions of $H$,

$$
H \varphi_{n}=2(n+1) \varphi_{n}, \quad n \geqslant 0 .
$$

We can show (see [GGT19]) that $\Pi$, the orthogonal projection on $\mathcal{E}$, is given by the formula

$$
(\Pi u)(z)=\frac{1}{\pi} e^{-\frac{|z|^{2}}{2}} \int_{\mathbb{C}} e^{\bar{w} z-\frac{|w|^{2}}{2}} u(w) d L(w),
$$


where $L$ stands for Lebesgue measure on $\mathbb{C}$.

Recall (see $(2.3)$ ) that for $s \geqslant 0$, the weighted Lebesgue space $L^{2, s}$ is defined by

$$
L^{2, s}=\left\{u \in L^{2}(\mathbb{C}),\langle z\rangle^{s} u \in L^{2}(\mathbb{C})\right\}, \quad\langle z\rangle=\left(1+|z|^{2}\right)^{1 / 2}
$$

and $L_{\mathcal{E}}^{2, s}=L^{2, s} \cap \mathcal{E}$. For $s \geqslant 0$, we define the harmonic Sobolev spaces by

$$
\mathbb{H}^{s}=\left\{u \in L^{2}(\mathbb{C}), H^{s / 2} u \in L^{2}(\mathbb{C})\right\} \cap \mathcal{E},
$$

equipped with the natural norm $\|u\|_{\mathbb{H}^{s}}=\left\|H^{s / 2} u\right\|_{L^{2}(\mathbb{C})}$. Then by [GGT19, Lemma C.1], we have $\mathbb{H}^{s}=L_{\mathcal{E}}^{2, s}$ with the equivalence of norms

$$
c\left\|\langle z\rangle^{s} u\right\|_{L^{2}(\mathbb{C})} \leqslant\|u\|_{\mathbb{H}^{s}} \leqslant C\left\|\langle z\rangle^{s} u\right\|_{L^{2}(\mathbb{C})}, \quad \forall u \in L_{\mathcal{E}}^{2, s} .
$$

Recall the hypercontractivity estimates (see [Car91] or [ST21, Lemma A.2] for the bounds without the optimal constants which will be enough for our purpose) : for all $1 \leqslant p \leqslant q \leqslant+\infty$ and $u \in \widetilde{\mathcal{E}}$

$$
\left(\frac{q}{2 \pi}\right)^{1 / q}\|u\|_{L^{q}(\mathbb{C})} \leqslant\left(\frac{p}{2 \pi}\right)^{1 / p}\|u\|_{L^{p}(\mathbb{C})} .
$$

In this paper $c, C>0$ denote universal constants the value of which may change from line to line.

\section{Study of the linear LLL equation}

\subsection{Global existence}

To solve equation (2.1) we find a fixed point in a ball of $\mathcal{E}$ to

$$
F: u \longmapsto e^{-i \delta t H} u_{0}-i \int_{0}^{t} e^{-i \delta(t-s) H}(\Pi(W u)(s)) d s .
$$

Let us sketch the proof: since $e^{i \tau H}$ is unitary in $L^{2}$, we have

$$
\begin{aligned}
\|F(u)(t)\|_{L^{2}} & \leqslant\left\|u_{0}\right\|_{L^{2}}+\int_{0}^{t}\|\Pi(W u)(s)\|_{L^{2}} d s \\
& \leqslant\left\|u_{0}\right\|_{L^{2}}+C t \sup _{s \in[0, t]}\|u(s)\|_{L^{2}}\|W\|_{L^{\infty}},
\end{aligned}
$$

where we used the continuity of $\Pi$ in $L^{2}$ in the last line (for continuity results for $\Pi$ we refer to [GGT19, Proposition 3.1]). Contraction estimates are obtained similarly, and this gives a local in time solution. Globalization can be obtained by the Grönwall inequality since the equation is linear. The $L^{2}$ norm of a solution is a conserved quantity, since the potential $W$ is real valued.

If moreover $u_{0} \in L_{\mathcal{E}}^{2, s}$, we can prove the wellposedness in $L_{\mathcal{E}}^{2, s}$, thanks to the following lemma, which we quote for future reference:

LEMmA 3.1. - Let $W \in L^{\infty}(\mathbb{C})$ and $v \in L_{\mathcal{E}}^{2, s}$, then

$$
\left\|\langle z\rangle^{s} \Pi(W v)\right\|_{L^{2}(\mathbb{C})} \leqslant C\|W\|_{L^{\infty}(\mathbb{C})}\left\|\langle z\rangle^{s} v\right\|_{L^{2}(\mathbb{C})},
$$

and

$$
\left\|\langle z\rangle^{s} e^{i \tau H} v\right\|_{L^{2}}=\left\|\langle z\rangle^{s} v\right\|_{L^{2}} .
$$


Proof. - The bound (3.1) is a consequence of [GGT19, Proposition 3.1]. For (3.2), we first observe that for all $u \in \mathcal{E}=L_{\mathcal{E}}^{2,0}$, we have $e^{i \tau H} u(z)=e^{2 i \tau} u\left(e^{2 i \tau} z\right)$, as can be seen by testing on the complete family $\left(\varphi_{n}\right)_{n \geqslant 0}$. Then (3.2) follows from the change of variables $z \mapsto e^{-2 i \tau} z$.

\subsection{Bounds on Sobolev norms: proof of Theorem 2.2}

Now that equation (2.1) is well-posed, let us inspect the behaviour of the norms of the solutions. For this we need a result, which is an consequence of [ST21, Lemma 2.1]:

Lemma 3.2. - Let $k \in \mathbb{N}$ and let $W \in \mathcal{C}^{k}\left(\mathbb{R} \times \mathbb{R}^{2}, \mathbb{R}\right)$ be a real valued function. Assume that $u \in L_{\mathcal{E}}^{2, k}$ satisfies

$$
i \partial_{t} u-\delta H u=\Pi(W u) .
$$

Then

$$
\begin{aligned}
\frac{d}{d t} \int_{\mathbb{C}}|z|^{2 k}|u(t, z)|^{2} d L(z) & \\
= & -2 \sum_{j=1}^{k}(-1)^{j}\left(\begin{array}{c}
k \\
j
\end{array}\right) \mathfrak{I m} \int_{\mathbb{C}} z^{k} \bar{z}^{k-j}|u(t, z)|^{2}\left(\partial_{z}^{j} W(t, z)\right) d L(z) .
\end{aligned}
$$

Proof. - We compute

$$
\begin{aligned}
\frac{d}{d t} \int_{\mathbb{C}}|z|^{2 k}|u|^{2} d L & =2 \mathfrak{R e} \int_{\mathbb{C}}|z|^{2 k} \bar{u} \partial_{t} u d L \\
& =2 \mathfrak{I m} \int_{\mathbb{C}}|z|^{2 k} \bar{u} \Pi(W u) d L+2 \delta \mathfrak{I m} \int_{\mathbb{C}}|z|^{2 k} \bar{u} H u d L .
\end{aligned}
$$

Let us first show that $\mathfrak{I m} \int_{\mathbb{C}}|z|^{2 k} \bar{u} H u d L=0$. Since $H=-4 \partial_{z} \partial_{\bar{z}}+|z|^{2}$, it remains to show that $\mathfrak{I m} \int_{\mathbb{C}}|z|^{2 k} \bar{u} \partial_{z} \partial_{\bar{z}} u d L=0$. Write $u(z)=f(z) e^{-\frac{1}{2}|z|^{2}}$, then

$$
\begin{aligned}
\mathfrak{I m} \int_{\mathbb{C}}|z|^{2 k} \bar{u} \partial_{z} \partial_{\bar{z}} u d L & =\mathfrak{I m} \int_{\mathbb{C}}|z|^{2 k} \bar{f} e^{-\frac{1}{2}|z|^{2}} \partial_{z} \partial_{\bar{z}}\left(f e^{-\frac{1}{2}|z|^{2}}\right) d L \\
& =-\frac{1}{2} \mathfrak{I m} \int_{\mathbb{C}}|z|^{2 k} \bar{f}\left(f+z \partial_{z} f-\frac{1}{2}|z|^{2} f\right) e^{-|z|^{2}} d L \\
& =-\frac{1}{2} \mathfrak{I m} \int_{\mathbb{C}} z^{k+1} \bar{z}^{k} \bar{f}\left(\partial_{z} f\right) e^{-|z|^{2}} d L \\
& =0,
\end{aligned}
$$

by integrating by parts, hence the result. To complete the proof, we apply [ST21, Lemma 2.1].

We are now able to prove Theorem 2.2. By linearity, it is enough to consider the case $\left\|\langle z\rangle^{k} u_{0}\right\|_{L^{2}(\mathbb{C})}=1$. We use the identity (3.3). Then, since $\left\|\partial_{z}^{j} W\right\|_{L^{\infty}(\mathbb{C})} \leqslant C_{0}$ for all $1 \leqslant j \leqslant k$, we deduce by Hölder

$$
\begin{aligned}
\frac{d}{d t} \int_{\mathbb{C}}|z|^{2 k}|u|^{2} d L & \leqslant C C_{0} \int_{\mathbb{C}}\langle z\rangle^{2 k-1}|u|^{2} d L \\
& \leqslant C C_{0}\left(\int_{\mathbb{C}}\langle z\rangle^{2 k}|u|^{2} d L\right)^{1-\frac{1}{2 k}}\left(\int_{\mathbb{C}}|u|^{2} d L\right)^{\frac{1}{2 k}},
\end{aligned}
$$


therefore, using the conservation of the mass,

$$
\frac{d}{d t}\left\|\langle z\rangle^{k} u\right\|_{L^{2}(\mathbb{C})}^{2} \leqslant C C_{0}\left\|\langle z\rangle^{k} u\right\|_{L^{2}(\mathbb{C})}^{2-\frac{1}{k}},
$$

which in turn implies, by time integration,

$$
\left\|\langle z\rangle^{k} u(t)\right\|_{L^{2}(\mathbb{C})} \leqslant\left(\left\|\langle z\rangle^{k} u_{0}\right\|_{L^{2}(\mathbb{C})}^{1 / k}+C C_{0}|t|\right)^{k} \leqslant C\left(1+C_{0}|t|\right)^{k},
$$

hence the result when $k$ is an integer. The general case follows by interpolation.

\subsection{Bounds on Sobolev norms: proof of Theorem 2.4}

Proof. - The proof is similar, excepted that now we have better controls on $W$.

(i) Again, by interpolation, it is enough to consider the case $s=k$ is an integer. By (3.3) we have

which implies the result by time integration :

$$
\frac{d}{d t} \int_{\mathbb{C}}|z|^{2 k}|u|^{2} d L \leqslant C C_{1} \int_{\mathbb{C}}|u|^{2} d L
$$

$$
\left\|\langle z\rangle^{k} u\right\|_{L^{2}(\mathbb{C})}^{2} \leqslant\left\|\langle z\rangle^{k} u_{0}\right\|_{L^{2}(\mathbb{C})}^{2}+C C_{1}|t|\left\|u_{0}\right\|_{L^{2}(\mathbb{C})}^{2}
$$

(ii) We assume that the stronger condition (2.12) holds. We use here interpolation theory for linear operators. Fix $s>0, \epsilon>0$ and set $k \in \mathbb{N}$ such that $s / k<\epsilon$. Then from (i) we have

$$
\left\|\langle z\rangle^{k} u(t)\right\|_{L^{2}(\mathbb{C})} \leqslant C\langle t\rangle^{1 / 2}\left\|\langle z\rangle^{k} u_{0}\right\|_{L^{2}(\mathbb{C})} .
$$

Next, the $L^{2}$-conservation yields $\|u(t)\|_{L^{2}(\mathbb{C})} \leqslant\left\|u_{0}\right\|_{L^{2}(\mathbb{C})}$. Then by interpolation, we get that for all $0 \leqslant \theta \leqslant 1$

$$
\left\|\langle z\rangle^{\theta k} u(t)\right\|_{L^{2}(\mathbb{C})} \leqslant C^{\theta}\langle t\rangle^{\theta / 2}\left\|\langle z\rangle^{\theta k} u_{0}\right\|_{L^{2}(\mathbb{C})} .
$$

The result then follows by taking $\theta=s / k$.

\subsection{Growth of Sobolev norms: proof of Theorem 2.3}

Let us define the magnetic translations by the formula

$$
R_{\alpha}:(u, v)(z) \mapsto\left(u(z+\alpha) e^{\frac{1}{2}(\bar{z} \alpha-z \bar{\alpha})}, v(z+\alpha) e^{\frac{1}{2}(\bar{z} \alpha-z \bar{\alpha})}\right), \quad \alpha \in \mathbb{C},
$$

as well as the space rotations

$$
L_{\theta}:(u, v)(z) \mapsto\left(u\left(e^{i \theta} z\right), v\left(e^{i \theta} z\right)\right), \quad \theta \in \mathbb{T} .
$$

As it can be checked on the $\left(\varphi_{n}\right)_{n \geqslant 0}$, we have $e^{i t H}=e^{2 i t} L_{2 t}$ for all $t \in \mathbb{R}$. Now we refer to [ST21, Section 1.7.2]. The system

$$
\left\{\begin{array}{l}
i \partial_{t} u-\delta H u=\Pi\left(|v|^{2} u\right), \quad(t, z) \in \mathbb{R} \times \mathbb{C} \\
i \partial_{t} v-\delta H v=-\Pi\left(|u|^{2} v\right) \\
u(0, z)=u_{0}(z), v(0, z)=v_{0}(z),
\end{array}\right.
$$


admits the following explicit solutions:

$$
\begin{aligned}
(u, v) & =\left(e^{-i \lambda t} e^{-i \delta t H} R_{\alpha t} U, e^{-i \mu t} e^{-i \delta t H} R_{\alpha t} V\right) \\
& =\left(e^{-i(\lambda+2 \delta) t} L_{-2 \delta t} R_{\alpha t} U, e^{-i(\mu+2 \delta) t} L_{-2 \delta t} R_{\alpha t} V\right),
\end{aligned}
$$

with

$$
U=\sqrt{\epsilon}\left(\frac{1}{2} \varphi_{0}+i \frac{\sqrt{3}}{2} \varphi_{1}\right), \quad V=\sqrt{\epsilon}\left(\frac{1}{2} \varphi_{0}-i \frac{\sqrt{3}}{2} \varphi_{1}\right)
$$

and

$$
\lambda=\frac{7 \epsilon}{32 \pi}, \quad \mu=-\frac{7 \epsilon}{32 \pi}, \quad \alpha=\frac{\sqrt{3}}{32 \pi} \epsilon .
$$

It remains to check that $W:=|v|^{2}$ and $u$ satisfy the assumptions and the conclusions of Theorem 2.3.

On the one hand, for all $t \in \mathbb{R},\|u(t)\|_{L^{2}}=\sqrt{\epsilon}$, and for $s>0$,

$$
\left\|\langle z\rangle^{s} u_{0}\right\|_{L^{2}}=\left\|\langle z\rangle^{s} U\right\|_{L^{2}} \leqslant c_{s} \sqrt{\epsilon} .
$$

Let us prove that there exists $c_{s}>0$ such that for all $t \in \mathbb{R}$

$$
\left\|\langle z\rangle^{s} u(t)\right\|_{L^{2}} \geqslant c_{s} \sqrt{\epsilon}\langle\epsilon t\rangle^{s} .
$$

We have

$$
\left\|\langle z\rangle^{s} u(t)\right\|_{L^{2}}^{2}=\left\|\langle z\rangle^{s} R_{\alpha t} U\right\|_{L^{2}}^{2}=\left\|\langle z-\alpha t\rangle^{s} U\right\|_{L^{2}}^{2}=\int_{\mathbb{C}}\left(1+|z-\alpha t|^{2}\right)^{s}|U(z)|^{2} d L(z) .
$$

Therefore,

$$
\left\|\langle z\rangle^{s} u(t)\right\|_{L^{2}}^{2} \geqslant \frac{1}{2} \int_{\mathbb{C}}\left(1+|z-\alpha t|^{2 s}\right)|U(z)|^{2} d L(z) .
$$

- By the triangle inequality we have

$$
|\alpha t|^{2 s} \leqslant(|z-\alpha t|+|z|)^{2 s} \leqslant 4^{s}\left(|z-\alpha t|^{2 s}+|z|^{2 s}\right),
$$

which in turn implies

$$
|z-\alpha t|^{2 s} \geqslant 4^{-s}|\alpha t|^{2 s}-|z|^{2 s} .
$$

As a consequence, by (3.4) and (3.6)

$$
\begin{aligned}
\left\|\langle z\rangle^{s} u(t)\right\|_{L^{2}}^{2} & \geqslant c|\alpha t|^{2 s} \int_{\mathbb{C}}|U(z)|^{2} d L(z)-C \int_{\mathbb{C}}|z|^{2 s}|U(z)|^{2} d L(z) \\
& \geqslant\left(c|\alpha t|^{2 s}-C\right) \epsilon \\
& \geqslant\left(c|\epsilon t|^{2 s}-C\right) \epsilon,
\end{aligned}
$$

where the constants $c, C>0$ have varied from line to line. From (3.7) we deduce that there exists $C_{0}>0$ such that if $|\epsilon t|>C_{0}$ we have

$$
\left\|\langle z\rangle^{s} u(t)\right\|_{L^{2}} \geqslant c\langle\epsilon t\rangle^{s} \sqrt{\epsilon} .
$$


- In the regime $|\epsilon t| \leqslant C_{0}$, we use the inequality (3.6) to write

$$
\left\|\langle z\rangle^{s} u(t)\right\|_{L^{2}} \geqslant c\|U\|_{L^{2}}=c \sqrt{\epsilon} \geqslant c\langle\epsilon t\rangle^{s} \sqrt{\epsilon} .
$$

As a consequence, we have proven (3.5).

On the other hand, we have the explicit expression

$$
W(t, z)=\frac{\epsilon}{4 \pi}\left|1-i \sqrt{3}\left(e^{-2 i \delta t} z+\alpha t\right)\right|^{2} e^{-\left|e^{-2 i \delta t} z+\alpha t\right|^{2}}=\left|L_{-2 \delta t} R_{\alpha t} V(z)\right|^{2} .
$$

Therefore we have

$$
\|W\|_{L^{1}}=\left\|L_{-2 \delta t} R_{\alpha t} V\right\|_{L^{2}}^{2}=\|V\|_{L^{2}}^{2}=\epsilon
$$

and from (2.15) we have

$$
\|W\|_{L^{\infty}}=\left\|L_{-2 \delta t} R_{\alpha t} V\right\|_{L^{\infty}}^{2}=\|V\|_{L^{\infty}}^{2} \leqslant \epsilon .
$$

Moreover, from Lemma C.2, we deduce

$$
\left\|\partial_{\bar{z}}^{j} \partial_{z}^{k} W\right\|_{L^{\infty}(\mathbb{C})}=\left\|\partial_{\bar{z}}^{j} \partial_{z}^{k}\left(\left|L_{-2 \delta t} R_{\alpha t} V\right|^{2}\right)\right\|_{L^{\infty}(\mathbb{C})} \leqslant C_{j k}\left\|L_{-2 \delta t} R_{\alpha t} V\right\|_{L^{\infty}}^{2} \leqslant \epsilon C_{j k},
$$

which was the claim.

\section{Proof of Theorem 1.2}

\subsection{Some notations}

For $0 \leqslant \tau<1$ we set $\rho(\tau)=\frac{1}{2(1-\tau)}>0$ and we define the operator $\widetilde{H}_{\tau}=(H+1)^{\rho(\tau)}$, where $H$ is the harmonic oscillator defined by

$$
H=-4 \partial_{z} \partial_{\bar{z}}+|z|^{2}
$$

We then define the family of Hilbert spaces $\left(\widetilde{\mathbb{H}}_{\tau}^{s}\right)_{s \geqslant 0}$ by

$$
\widetilde{\mathbb{H}}_{\tau}^{s}=\left\{u \in L^{2}(\mathbb{C}), \widetilde{H}^{s / 2} u \in L^{2}(\mathbb{C})\right\} \cap \mathcal{E}, \quad \widetilde{\mathbb{H}}_{\tau}^{0}=\mathcal{E} .
$$

Recall that, by [GGT19, Lemma C.1], we have

$$
c\left\|\langle z\rangle^{s \rho(\tau)} u\right\|_{L^{2}(\mathbb{C})} \leqslant\|u\|_{\widetilde{\mathbb{H}}_{\tau}^{s}} \leqslant C\left\|\langle z\rangle^{s \rho(\tau)} u\right\|_{L^{2}(\mathbb{C})}, \quad\langle z\rangle=\left(1+|z|^{2}\right)^{1 / 2} .
$$

Observe also that

$$
\widetilde{\mathbb{H}}_{\tau}^{s}=\mathbb{H}^{s \rho(\tau)}
$$

where $\mathbb{H}^{\sigma}$ stands for the harmonic Sobolev space based on the harmonic oscillator $H$, and we have

$$
c\left\|\langle z\rangle^{s} u\right\|_{L^{2}(\mathbb{C})} \leqslant\|u\|_{\mathbb{H}^{s}} \leqslant C\left\|\langle z\rangle^{s} u\right\|_{L^{2}(\mathbb{C})} .
$$

In the sequel, in order to alleviate notations, we simply write $\widetilde{H}=\widetilde{H}_{\tau}$ and $\widetilde{\mathbb{H}}^{s}=\widetilde{\mathbb{H}}_{\tau}^{s}$. 


\subsection{Definition of the operator $\mathscr{L}(t)$}

Define the potential $W_{0}(t, z)$ as follows:

$$
\begin{aligned}
V & =\sqrt{\epsilon}\left(\frac{1}{2} \varphi_{0}-i \frac{\sqrt{3}}{2} \varphi_{1}\right), \\
W_{0}(t, z) & =\left|R_{\alpha t} V(z)\right|^{2}=\frac{\epsilon}{4 \pi}|1-i \sqrt{3}(z+\alpha t)|^{2} e^{-|z+\alpha t|^{2}}, \\
\alpha & =\frac{\sqrt{3}}{32 \pi} \epsilon
\end{aligned}
$$

and with Lemma C.2, we show that all the derivatives of $W_{0}$ are bounded uniformly in $t \in \mathbb{R}$ :

$$
\begin{aligned}
\| \partial_{\bar{z}}^{j} \partial_{z}^{k} W_{0}(t) & \|_{L^{\infty}(\mathbb{C})} \\
& =\left\|\partial_{\bar{z}}^{j} \partial_{z}^{k}\left(\left|R_{\alpha t} V\right|^{2}\right)\right\|_{L^{\infty}(\mathbb{C})} \leqslant C_{j k}\left\|R_{\alpha t} V\right\|_{L^{\infty}}^{2}=C_{j k}\|V\|_{L^{\infty}}^{2} \leqslant \epsilon C_{j k} .
\end{aligned}
$$

Now we define the mapping

$$
\begin{aligned}
& \mathscr{L}(t): \widetilde{\mathbb{H}}^{s} \longrightarrow \widetilde{\mathbb{H}}^{s} \\
& u \longmapsto e^{-i t \widetilde{H}} \Pi\left(W_{0}(t) e^{i t \widetilde{H}} u\right)=e^{-i t(H+1)^{\rho}} \Pi\left(W_{0}(t) e^{i t(H+1)^{\rho}} u\right),
\end{aligned}
$$

and we consider the initial value problem

$$
\left\{\begin{array}{l}
i \partial_{t} u=(\widetilde{H}+\mathscr{L}(t)) u, \quad(t, z) \in \mathbb{R} \times \mathbb{C}, \\
u(t)_{\mid t=t_{0}}=u_{0} \in \widetilde{\mathbb{H}}^{s} .
\end{array}\right.
$$

\subsection{Verification of Assumption 1.1}

We now prove that $\mathscr{L}(t)$ satisfies the required properties.

Proof of Assumption 1.1. -

(i) Let us check that $\mathscr{L} \in \mathcal{C}_{b}\left(\mathbb{R}, \mathcal{L}\left(\widetilde{\mathbb{H}}^{s}\right)\right)$, with norm $\|\mathscr{L}(t)\|_{\mathcal{L}\left(\widetilde{\mathbb{H}}^{s}\right)} \leqslant C_{s} \epsilon$. First, by (4.1) it is equivalent to show that $\mathscr{L} \in \mathcal{C}_{b}\left(\mathbb{R}, \mathcal{L}\left(\mathbb{H}^{s}\right)\right)$. Then, since $e^{i t(H+1)^{\rho}}$ is unitary in $\mathbb{H}^{s}$, and by (4.2)

$$
\begin{aligned}
\left\|e^{-i t(H+1)^{\rho}} \Pi\left(W_{0}(t) e^{i t(H+1)^{\rho}} u\right)\right\|_{\mathbb{H}^{s}} & =\left\|\Pi\left(W_{0}(t) e^{i t(H+1)^{\rho}} u\right)\right\|_{\mathbb{H}^{s}} \\
& \leqslant C\left\|\langle z\rangle^{s} \Pi\left(W_{0}(t) e^{i t(H+1)^{\rho}} u\right)\right\|_{L^{2}(\mathbb{C})} .
\end{aligned}
$$

Next, by (3.1) and (3.2)

$$
\begin{aligned}
\left\|\langle z\rangle^{s} \Pi\left(W_{0}(t) e^{i t(H+1)^{\rho}} u\right)\right\|_{L^{2}(\mathbb{C})} & \leqslant C\left\|W_{0}(t)\right\|_{L^{\infty}(\mathbb{C})}\left\|\langle z\rangle^{s} e^{i t(H+1)^{\rho}} u\right\|_{L^{2}(\mathbb{C})} \\
& =C\left\|W_{0}(t)\right\|_{L^{\infty}(\mathbb{C})}\left\|\langle z\rangle^{s} u\right\|_{L^{2}(\mathbb{C})} \\
& \leqslant C\left\|W_{0}(t)\right\|_{L^{\infty}(\mathbb{C})}\|u\|_{\mathbb{H}^{s}} .
\end{aligned}
$$


Recall that $W_{0}(t)=\left|R_{\alpha t} V\right|^{2}$, where $V \in \mathcal{E}$, then $\left\|W_{0}(t)\right\|_{L^{\infty}(\mathbb{C})}=\|V\|_{L^{\infty}}^{2} \leqslant C \epsilon$. Putting all the previous estimates together, we obtain

$$
\left\|e^{-i t(H+1)^{\rho}} \Pi\left(W_{0}(t) e^{i t(H+1)^{\rho}} u\right)\right\|_{\mathbb{H}^{s}} \leqslant C_{s} \epsilon\|u\|_{\mathbb{H}^{s}},
$$

hence the announced bound. The time-continuity of $\mathscr{L}$ follows from the previous estimates together with the continuity of the translations for the Lebesgue measure and the fact that $e^{i t \widetilde{H}} \in \mathcal{C}_{b}\left(\mathbb{R}, \mathcal{L}\left(\widetilde{\mathbb{H}}^{s}\right)\right)$.

(ii) The symmetry of $\mathscr{L}$, w.r.t. the scalar product of $\widetilde{\mathbb{H}}^{0}=\mathcal{E}$, is a consequence of the symmetry of $\Pi$, the conjugation by the unitary operator $e^{i t(H+1)^{\rho}}$ and the fact that $W_{0}$ is a real valued function.

(iii) Let us check that $[\widetilde{H}, \mathscr{L}(t)]$ is $\widetilde{H}^{\tau}$-bounded. By Lemma C.1, the operators $H$ and $\Pi$ commute, thus

$$
\begin{aligned}
{[\widetilde{H}, \mathscr{L}(t)] \widetilde{H}^{-\tau} } & =\left[(H+1)^{\rho}, \mathscr{L}(t)\right](H+1)^{-\rho \tau} \\
& =e^{-i t(H+1)^{\rho}} \Pi\left[(H+1)^{\rho}, W_{0}(t)\right](H+1)^{-\rho \tau} e^{i t(H+1)^{\rho}} .
\end{aligned}
$$

Recall that $\Pi$ is bounded in all the $\mathbb{H}^{s}$ spaces, as well as the operators $e^{-i t(H+1)^{\rho}}$.

- Case $s=0$. Let us first prove that $\Pi\left[(H+1)^{\rho}, W_{0}(t)\right](H+1)^{-\rho \tau}: \mathcal{E} \longrightarrow \mathcal{E}$ is bounded, uniformly in $t \in \mathbb{R}$. For that, we use the Weyl-Hörmander pseudodifferential calculus (we refer to [Hel84, Rob87] or to [Par10, Chapter 3] for a review of this theory). Denote by $z=x_{1}+i x_{2}, \xi=\xi_{1}+\xi_{2}$, and consider the metric

$$
d x_{1}^{2}+d x_{2}^{2}+\frac{d \xi_{1}^{2}+d \xi_{2}^{2}}{1+|z|^{2}+|\xi|^{2}} .
$$

The Planck function associated to the metric (4.6) is given by $h\left(x_{1}, x_{2}, \xi_{1}, \xi_{2}\right)=$ $\left(1+|z|^{2}+|\xi|^{2}\right)^{-1 / 2}$ and for $m \in \mathbb{R}$, the symbol class $S^{m}$ is

$$
\begin{aligned}
S^{m}=\left\{a \in \mathcal{C}^{\infty}\left(\mathbb{R}^{4} ; \mathbb{C}\right):\right. & \left|\partial_{x_{1}}^{\alpha_{1}} \partial_{x_{2}}^{\alpha_{2}} \partial_{\xi_{1}}^{\beta_{1}} \partial_{\xi_{2}}^{\beta_{2}} a\left(x_{1}, x_{2}, \xi_{1}, \xi_{2}\right)\right| \\
& \left.\leqslant C_{\alpha, \beta}\langle|z|+|\xi|\rangle^{m-\beta_{1}-\beta_{2}}, \quad \forall \alpha, \beta \in \mathbb{N}^{2}\right\} .
\end{aligned}
$$

For $a \in S^{m}$, we define its Weyl-quantization by the formula

$$
a^{w}(x, D) u(x)=\frac{1}{(2 \pi)^{2}} \int_{\mathbb{R}^{2}} \int_{\mathbb{R}^{2}} e^{i(x-y) \cdot \xi} a\left(\frac{x+y}{2}, \xi\right) u(y) d y d \xi, \quad u \in \mathscr{S}\left(\mathbb{R}^{2}\right) .
$$

First, using the functional calculus associated to the operator $H$, we obtain that $(H+1)^{\rho}$ is a pseudo-differential operator with symbol in $S^{2 \rho}$. By $(4.3), W_{0}(t) \in S^{0}$ uniformly in $t \in \mathbb{R}$, and therefore the commutator $\left[(H+1)^{\rho}, W_{0}(t)\right]$ is a pseudodifferential operator with symbol in $S^{2 \rho-1}$, which in turn implies that

$$
\left[(H+1)^{\rho}, W_{0}(t)\right](H+1)^{-\rho \tau}
$$

is a pseudo-differential operator with symbol in $S^{0}$ (because $2 \rho-1-2 \rho \tau=0$ ), hence it is bounded. 
- The proof in the general case $s \geqslant 0$ is similar.

(iv) From (4.4), a direct computation gives

$$
\begin{aligned}
\partial_{t} \mathscr{L}(t) & =e^{-i t \widetilde{H}} \Pi\left(\partial_{t} W_{0}(t)\right) e^{i t \widetilde{H}}-i e^{-i t \widetilde{H}} \Pi\left[\widetilde{H}, W_{0}(t)\right] e^{i t \widetilde{H}} \\
& =e^{-i t \widetilde{H}} \Pi\left(\partial_{t} W_{0}(t)\right) e^{i t \widetilde{H}}-i[\widetilde{H}, \mathscr{L}(t)] .
\end{aligned}
$$

From the expression of $W_{0}$, we deduce that $\sup _{t \in \mathbb{R}}\left\|\partial_{t}^{\ell} W_{0}(t)\right\|_{L^{\infty}(\mathbb{C})} \leqslant C_{\ell}$ for all $\ell \geqslant 0$. In particular, the first term in the right hand side of $(4.7)$ is bounded $\widetilde{\mathbb{H}}^{s} \longrightarrow \widetilde{\mathbb{H}}^{s}$. In item (iii) we have shown that $[\widetilde{H}, \mathscr{L}(t)]: \widetilde{\mathbb{H}}^{s} \longrightarrow \widetilde{\mathbb{H}}^{s-\tau}$ is bounded, uniformly in $t \in \mathbb{R}$. As a consequence, for all $s \geqslant 0, \mathscr{L} \in \mathcal{C}_{b}^{1}\left(\mathbb{R} ; \mathcal{L}\left(\widetilde{\mathbb{H}}{ }^{s} ; \widetilde{\mathbb{H}}^{s-\tau}\right)\right)$. The general case $\ell \geqslant 0$ is obtained by induction.

\subsection{Proof of Theorem 1.2}

Proof. - We are now ready to complete the proof of Theorem 1.2. Consider the problem (4.5) and for convenience, assume that $t_{0}=0$. By (4.4), the equation (4.5) is equivalent to

$$
\left\{\begin{array}{l}
i \partial_{t} v=\Pi\left(W_{0}(t) v\right), \quad(t, z) \in \mathbb{R} \times \mathbb{C} \\
v(0, \cdot)=u_{0} \in \widetilde{\mathbb{H}}^{s}=\mathbb{H}^{\rho s}
\end{array}\right.
$$

with the change of unknown $v=e^{i t(H+1)^{\rho}} u$. As a consequence we can directly apply the results of Section 2 (case $\delta=0$ ) to this model.

(i) The fact that $\|\mathscr{L}(t)\|_{\mathcal{L}\left(\widetilde{\mathbb{H}}^{s}\right)} \leqslant C_{s} \epsilon$ has already been shown in the previous paragraph.

(ii) For all $s \geqslant 0$, the problem (4.5) is globally well-posed, in $\widetilde{\mathbb{H}}^{s}$ by Theorem 2.1. The group property of $\mathcal{U}$ is a consequence of uniqueness, and its unitarity follows from the conservation of the $L^{2}$ norm.

(iii) The upper bound is given by Theorem 2.2 , namely, for all $t \in \mathbb{R}$

$$
\|u(t)\|_{\widetilde{\mathbb{H}}^{s}} \leqslant C\left\|\langle z\rangle^{\rho s} u(t)\right\|_{L^{2}(\mathbb{C})} \leqslant C\left\|\langle z\rangle^{\rho s} u_{0}\right\|_{L^{2}(\mathbb{C})}\langle\epsilon t\rangle^{\rho s} \leqslant C\left\|u_{0}\right\|_{\widetilde{\mathbb{H}}^{s}}\langle\epsilon t\rangle^{\rho s},
$$

where $\rho=\frac{1}{2(1-\tau)}$.

(iv) Consider the function $u_{0} \in \cap_{k \geqslant 1} L_{\mathcal{E}}^{2, k}=\cap_{k} \geqslant 1 \widetilde{\mathbb{H}}^{k}$ given by Theorem 2.3, (see paragraph 3.4) then $\left\|u_{0}\right\|_{\widetilde{\mathbb{H}}^{s}} \leqslant C \sqrt{\epsilon}$ and

$$
\|u(t)\|_{\widetilde{\mathbb{H}}^{s}} \geqslant c\left\|\langle z\rangle^{\rho s} u(t)\right\|_{L^{2}(\mathbb{C})} \geqslant c \sqrt{\epsilon}\langle\epsilon t\rangle^{\rho s} \geqslant c\left\|u_{0}\right\|_{\widetilde{\mathbb{H}}^{s}}\langle\epsilon t\rangle^{\rho s},
$$

hence the result.

Notice that the items (ii) and (iii) also directly follow from the general result [MR17, Theorem 1.5]. 


\section{Appendix A. A non-perturbative \& time-independent example}

In this section, we give another example of linear Schrödinger operator which yields unbounded dynamics, and which meets the assumptions (H0)-(H3) of [MR17]. This example differs from the one exhibited in Theorem 1.2 in two main aspects:

- it is a non-perturbative example: it is not a lower order perturbation of a time-independent elliptic differential operator;

- it is time-independent.

However, with a change of unknown, we can obtain a time-dependent perturbation of a constant coefficient self-adjoint elliptic operator, see Remark A.3 below.

This example is very simple, and that is why we decided to develop it here. Actually the mechanism involved in the norm inflation is the same as in Theorem 1.2: it is a traveling wave measured in a weighted $L^{2}$ space. Actually, our example is close to the one developed in [BGMR18, Appendix A], after change of variables.

On $L^{2}(\mathbb{R})$ we define the usual harmonic oscillator $H=-\partial_{x}^{2}+x^{2}$. For $0 \leqslant \tau<1$, we set $\rho(\tau)=\frac{1}{2(1-\tau)} \in[1 / 2, \infty)$. We define the operator $\widetilde{H}=(H+1)^{\rho(\tau)}$ and the scale of Hilbert spaces $\left(\widetilde{\mathbb{H}^{s}}\right)_{s \geqslant 0}$ by

$$
\widetilde{\mathbb{H}}^{s}=\left\{u \in L^{2}(\mathbb{C}), \widetilde{H}^{s / 2} u \in L^{2}(\mathbb{R})\right\}, \quad \widetilde{\mathbb{H}}^{0}=L^{2}(\mathbb{R}),
$$

endowed with the natural norm $\|u\|_{\widetilde{H}^{s}}:=\left\|\widetilde{H}^{s / 2} u\right\|_{L^{2}(\mathbb{R})}$. By [YZ04, Lemma 2.4], we have the following equivalence of norms

$$
\|u\|_{\widetilde{\mathbb{H}}^{s}} \equiv\left\|\langle x\rangle^{\rho s} u\right\|_{L^{2}(\mathbb{R})}+\left\|\left(-\partial_{x}^{2}\right)^{\rho s / 2} u\right\|_{L^{2}(\mathbb{R})} .
$$

Now, for $\epsilon>0$, we consider the problem,

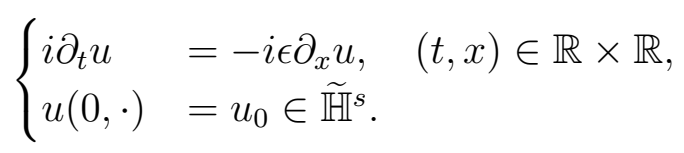

In this framework, we are able to prove the following result for the operator $i \epsilon \partial_{x}$ in the spaces $\widetilde{\mathbb{H}}^{s}$ :

LEMMA A.1. -

(i) One has $\left.i \epsilon \partial_{x} \in \mathcal{L}\left(\widetilde{\mathbb{H}}^{s+1 / \rho}, \widetilde{\mathbb{H}}^{s}\right)\right)$ for all $s \geqslant 0$, and

$$
\left\|i \epsilon \partial_{x}\right\|_{\mathcal{L}\left(\widetilde{\mathbb{H}}^{s+1 / \rho,}, \widetilde{\mathbb{H}}^{s}\right)} \leqslant C_{s} \epsilon .
$$

(ii) The operator $i \epsilon \partial_{x}$ is symmetric on $\widetilde{\mathbb{H}}^{1 / \rho}$ w.r.t. the scalar product of $\widetilde{\mathbb{H}}^{0}$,

$$
\int_{\mathbb{R}} \bar{v}\left(i \epsilon \partial_{x} u\right) d x=\int_{\mathbb{R}} u \overline{\left(i \epsilon \partial_{x} v\right)} d x, \quad \forall u, v \in \widetilde{\mathbb{H}}^{1 / \rho} .
$$

(iii) The operator $i \epsilon \partial_{x}$ is $\widetilde{H}^{\tau}$-bounded in the sense that $\left[i \epsilon \partial_{x}, \widetilde{H}\right] \widetilde{H}^{-\tau} \in \mathcal{L}\left(\widetilde{\mathbb{H}}^{s}\right)$ for all $s \geqslant 0$.

Therefore the operator $i \partial_{x}$ satisfies the assumptions (H0)-(H3) of [MR17].

On the other hand, we have the following elementary result:

Proposition A.2. - Let $s \geqslant 0$, then 
(i) The problem (A.2) is globally well-posed in $\widetilde{\mathbb{H}}^{s}$, and the solution is explicitly given by

$$
u(t, x)=u_{0}(x-\epsilon t) .
$$

(ii) The following bounds hold true : for all $u_{0} \in \widetilde{\mathbb{H}}^{s}$ and for all $t \in \mathbb{R}$

$$
c\langle\epsilon t\rangle^{\frac{s}{2(1-\tau)}}\left\|u_{0}\right\|_{\widetilde{\mathbb{H}}^{s}} \leqslant\|u(t)\|_{\widetilde{\mathbb{H}}^{s}} \leqslant C\langle\epsilon t\rangle^{\frac{s}{2(1-\tau)}}\left\|u_{0}\right\|_{\widetilde{\mathbb{H}}^{s}} .
$$

This result is directly obtained using (A.1) and the expression $\rho(\tau)=\frac{1}{2(1-\tau)}$.

Remark A.3. - Notice that the function $v(t)=e^{-i t \widetilde{H}} u(t)$ is solution to

$$
\left\{\begin{array}{l}
i \partial_{t} v-\widetilde{H} v=-i \epsilon\left(e^{-i t \widetilde{H}} \partial_{x} e^{i t \widetilde{H}}\right) v, \quad(t, x) \in \mathbb{R} \times \mathbb{R}, \\
v(0, \cdot)=v_{0}=u_{0} \in \widetilde{\mathbb{H}}^{s},
\end{array}\right.
$$

and satisfies the conclusions of Lemma A.1 and the bounds (A.3). This yields an example in the spirit of the one exhibited in Theorem 1.2, but in the present case, the perturbation is of order $1 / \rho \in(0,2]$ instead of being of order 0 .

Proof of Lemma A.1. - Item (i) is a direct consequence of (A.1) and (ii) is elementary.

(iii) It is convenient to introduce the Sobolev space based on the harmonic oscillator $(s \geqslant 0)$

$$
\mathbb{H}^{s}=\left\{u \in L^{2}(\mathbb{R}), H^{s / 2} u \in L^{2}(\mathbb{R})\right\}, \quad \mathbb{H}^{0}=L^{2}(\mathbb{R}) .
$$

Thanks to the pseudo-differential calculus associated to $H$ (see also paragraph 4.3), we first prove that for all $r>0$,

$$
\left[i \partial_{x},(H+1)^{r}\right](H+1)^{-r+\frac{1}{2}} \in \mathcal{L}\left(L^{2}(\mathbb{R})\right) .
$$

Here the symbol class $S^{m}$ reads

$$
S^{m}=\left\{a \in \mathcal{C}^{\infty}\left(\mathbb{R}^{2} ; \mathbb{C}\right):\left|\partial_{x}^{\alpha} \partial_{\xi}^{\beta} a(x, \xi)\right| \leqslant C_{\alpha, \beta}\langle|x|+|\xi|\rangle^{m-\beta}, \quad \forall \alpha, \beta \in \mathbb{N}\right\} .
$$

The symbol of $\left[i \partial_{x},(H+1)^{r}\right]$, modulo terms in $S^{2 r-1}$, is given by the formula

$$
\begin{aligned}
-i\left\{\xi,\left(x^{2}+\xi^{2}+1\right)^{r}\right\} & =-i \partial_{\xi}(\xi) \partial_{x}\left(\left(x^{2}+\xi^{2}+1\right)^{r}\right)+i \partial_{x}(\xi) \partial_{\xi}\left(\left(x^{2}+\xi^{2}+1\right)^{r}\right) \\
& =-i 2 r x\left(x^{2}+\xi^{2}+1\right)^{r-1} \in S^{2 r-1} .
\end{aligned}
$$

Since the symbol of $(H+1)^{-r+\frac{1}{2}}$ belongs to $S^{-2 r+1}$, we deduce (A.4).

Next, for $\rho>0$ and $s \geqslant 0$

$$
\begin{aligned}
(H+1)^{\frac{s}{2}} & {\left[i \partial_{x},(H+1)^{\rho}\right](H+1)^{-\rho+\frac{1}{2}}(H+1)^{-\frac{s}{2}} } \\
& =-\left[i \partial_{x},(H+1)^{\frac{s}{2}}\right](H+1)^{-\frac{s}{2}+\frac{1}{2}}+\left[i \partial_{x},(H+1)^{\frac{s}{2}+\rho}\right](H+1)^{-\left(\frac{s}{2}+\rho\right)+\frac{1}{2}},
\end{aligned}
$$

and by applying (A.4) twice, we deduce that for all $s \geqslant 0$

$$
\left[i \partial_{x},(H+1)^{\rho}\right](H+1)^{-\rho+\frac{1}{2}} \in \mathcal{L}\left(\mathbb{H}^{s}\right) .
$$

Finally recall that $\widetilde{H}=(H+1)^{\rho}$, thus (A.5) is equivalent to

$$
\left[i \partial_{x}, \widetilde{H}\right] \widetilde{H}^{-\tau} \in \mathcal{L}\left(\widetilde{\mathbb{H}}^{s}\right)
$$


since $\tau=1-1 /(2 \rho)$, which was the claim.

\section{Appendix B. On the reducibility of the linear LLL equation}

We state here a reducibility result for the linear LLL equation. It turns out that the abstract reducibility result obtained in [GT11] can be applied to this model, which is close in many aspects to the usual 1D cubic quantum harmonic oscillator with time-dependent potential. We consider the linear equation

$$
\left\{\begin{array}{l}
i \partial_{t} u-\delta H u=\epsilon \Pi(W(t \omega, z) u), \quad(t, z) \in \mathbb{R} \times \mathbb{C}, \\
u(0, z)=u_{0}(z),
\end{array}\right.
$$

where $\delta \neq 0$, where $\epsilon>0$ is small and where the parameter $\omega \in[0,2 \pi)^{n}$ is the frequency vector, for some given $n \geqslant 1$. Up to a rescaling, we can assume that $\delta=1$. We assume in the sequel that the potential

$$
\begin{aligned}
W: \mathbb{T}^{n} \times \mathbb{C} & \longrightarrow \mathbb{R} \quad \mathbb{T}^{n}:=(\mathbb{R} / 2 \pi \mathbb{Z})^{n} \\
(\theta, z) & \longmapsto W(\theta, z),
\end{aligned}
$$

is analytic in $\theta$ on $|\operatorname{Im} \theta|<\tau$ for some $\tau>0$, and $\mathcal{C}^{2}$ in $x, y$ (where $z=x+i y$ ), and we suppose moreover that there exists $\gamma>0$ and $C>0$ so that for all $\theta \in \mathbb{T}^{n}$ and $z \in \mathbb{C}$

$$
|W(\theta, z)| \leqslant C\langle z\rangle^{-\gamma}, \quad\left|\partial_{z}^{j} \partial_{\bar{z}}^{\ell} W(\theta, z)\right| \leqslant C
$$

for any $0 \leqslant j, \ell \leqslant 1$.

When $\omega=0$, all the solutions to (B.1) are almost periodic in time. This can be proved by constructing a Hilbertian basis ${ }^{(1)}\left(\psi_{k}\right)_{k \geqslant 0}$ of $\mathcal{E}$ composed of eigenfunctions of the operator $u \mapsto H u+\epsilon \Pi(W(0, z) u)$, such that

$$
H \psi_{k}+\epsilon \Pi\left(W(0, z) \psi_{k}\right)=\lambda_{k} \psi_{k}, \quad k \geqslant 0 .
$$

Then (B.1) can be solved by

$$
u(t, z)=\sum_{k=0}^{+\infty} c_{k} e^{-i t \lambda_{k}} \psi_{k}(z), \quad u_{0}(z)=\sum_{k=0}^{+\infty} c_{k} \psi_{k}(z),
$$

which shows that any solution to (B.1) is an infinite superposition of periodic functions, hence it is an almost-periodic function in time.

For $\omega \neq 0$, the reducibility theory addresses the question if, by the means of a time quasi-periodic transformation, one can reduce to the previous case. It turns out that for (B.1), it is the case for a large set of values $\omega \in \Lambda_{\epsilon}$ :

Theorem B.1. - Assume that $W$ satisfies (B.2). Then there exists $\epsilon_{0}$ such that for all $0 \leqslant \epsilon<\epsilon_{0}$ there exists a set $\Lambda_{\epsilon} \subset[0,2 \pi)^{n}$ of positive measure and asymptotically full measure: Meas $\left(\Lambda_{\epsilon}\right) \rightarrow(2 \pi)^{n}$ as $\epsilon \rightarrow 0$, such that for all $\omega \in \Lambda_{\epsilon}$, the linear equation (B.1) reduces, in $\mathcal{E}$, to a linear equation with constant coefficients.

${ }^{(1)}$ Such a Hilbertian basis exists, since $u \mapsto H u+\epsilon \Pi(W(0, z) u)$ is a self-adjoint operator with compact resolvent in $\mathcal{E}$. 
We refer to [GT11, Theorem 7.1] for a more precise statement, giving in particular more information on the transformation.

Assume that $(\theta, z) \mapsto V(\theta, z)$ is analytic in $\theta$ on $|\operatorname{Im} \theta|<\tau$, that $V(\theta, \cdot) \in \mathcal{E}$ for all $\theta \in \mathbb{T}^{n}$, and satisfies, for some $\gamma>0$, the bound $|V(\theta, z)| \leqslant C\langle z\rangle^{-\gamma}$ uniformly in $\theta \in \mathbb{T}^{n}$. Then, by Lemma C.2, $W=|V|^{2}$ satisfies (B.2). Such a potential even satisfies $\left|\partial_{z}^{j} \partial_{\bar{z}}^{\ell} W(\theta, z)\right| \leqslant C$ for all $k, \ell \in \mathbb{N}$ (without additional assumptions on $V \in \mathcal{E})$.

We also have the following result on the dynamics of the solutions of (B.1) :

Corollary B.2. - Assume that $W$ is $\mathcal{C}^{\infty}$ in $x, y$ with all its derivatives bounded and satisfying (B.2). Let $s \geqslant 0$ and $u_{0} \in \mathbb{H}^{s}$. Then there exists $\epsilon_{0}>0$ so that for all $0<\epsilon<\epsilon_{0}$ and $\omega \in \Lambda_{\epsilon}$, there exists a unique solution $u \in \mathcal{C}\left(\mathbb{R} ; \mathbb{H}^{s}\right)$ of (B.1) so that $u(0)=u_{0}$. Moreover, $u$ is almost-periodic in time and we have the bounds

$$
(1-\epsilon C)\left\|u_{0}\right\|_{\mathbb{H}^{s}} \leqslant\|u(t)\|_{\mathbb{H}^{s}} \leqslant(1+\epsilon C)\left\|u_{0}\right\|_{\mathbb{H}^{s}}, \quad \forall t \in \mathbb{R},
$$

for some $C=C(s, \omega)$.

The result of Theorem B.1 can also be formulated in term of the Floquet operator. Consider the Floquet Hamiltonian operator, defined on $\mathcal{E} \otimes L^{2}\left(\mathbb{T}^{n}\right)$ by

$$
K:=i \sum_{k=1}^{n} \omega_{k} \partial_{\theta_{k}}+H+\epsilon \Pi(W(\theta, z) \cdot),
$$

then we can state

Corollary B.3. - Assume that $W$ satisfies (B.2). There exists $\epsilon_{0}>0$ so that for all $0<\epsilon<\epsilon_{0}$ and $\omega \in \Lambda_{\epsilon}$, the spectrum of the Floquet operator $K$ is pure point.

We refer to [GT11, Section 7], where similar results are proven for the 1D quantum harmonic oscillator.

For the reducibility of the periodic Schrödinger equation, we refer to [EK09] and for the reducibility of the quantum harmonic oscillator in any dimension to [BGMR18, GP19, LW19] and we refer to [Bam17, Bam18, BM18] for the reducibility for 1-d operators with unbounded perturbations. For references on the theory of Floquet operators, see [Eli01, Wan08b].

Finally, let us mention that, concerning the nonlinear cubic LLL equation, the abstract KAM result of [GT11] was applied in [GGT19, Theorem 4.3] in order to show the existence of invariant torii, and that the result of [GIP09] was applied to show an almost global existence result for the cubic LLL equation. We refer to [GGT19, Section 4.2] for more details.

The arguments of [GT11, Section 7] can be directly applied to the equation (B.1), and we address the reader to this latter paper for the proofs of the previous results. Let us just sketch the idea : we expand $u$ and $\bar{u}$ on the basis given by the special Hermite functions

$$
u=\sum_{j \geqslant 0} c_{j} \varphi_{j}, \quad \bar{u}=\sum_{j \geqslant 0} \bar{c}_{j} \bar{\varphi}_{j}
$$


Then equation (B.1) reads as an autonomous Hamiltonian system in an extended phase space

$$
\begin{cases}\dot{c}_{j}=-2 i(j+1) c_{j}-i \epsilon \partial_{\bar{c}_{j}} Q(\theta, c, \bar{c}) & j \geqslant 0 \\ \dot{\bar{c}}_{j}=2 i(j+1) \bar{c}_{j}+i \epsilon \partial_{c_{j}} Q(\theta, c, \bar{c}) & j \geqslant 0 \\ \dot{\theta}_{j}=\omega_{j} & j=0, \ldots, n \\ \dot{Y}_{j}=-\epsilon \partial_{\theta_{j}} Q(\theta, z, \bar{z}) & j=0, \ldots, n\end{cases}
$$

where $Q$ is a quadratic functional in $(c, \bar{c})$ given by

$$
Q(\theta, c, \bar{c})=\int_{\mathbb{C}} W(\theta, z)\left(\sum_{j \geqslant 0} c_{k} \varphi_{k}(z)\right) \overline{\left(\sum_{j \geqslant 0} c_{j} \varphi_{j}(z)\right)} d L(z),
$$

and the Hamiltonian of the system (B.3) is

$$
\sum_{j=1}^{n} \omega_{j} Y_{j}+2 \sum_{j \geqslant 0}(j+1) c_{j} \bar{c}_{j}+Q(\theta, c, \bar{c}) .
$$

Then, we can check that (B.4) satisfies the assumptions of [GT11, Theorem 7.1]. The dispersive estimate $\left\|\varphi_{n}\right\|_{L^{\infty}(\mathbb{C})} \leqslant C n^{-1 / 4}$ satisfied by the $\left(\varphi_{n}\right)_{n \geqslant 0}$, is the key ingredient which allows to follow the lines of [GT11, Section 7].

\section{Appendix C. Some technical results}

Lemma C.1. - The operators $H$ and $\Pi$ commute.

Proof. - Recall that

$$
[\Pi u](z)=\frac{1}{\pi} e^{-\frac{|z|^{2}}{2}} \int_{\mathbb{C}} e^{\bar{w} z-\frac{|w|^{2}}{2}} u(w) d L(w),
$$

and that

$$
H=-4 \partial_{z} \partial_{\bar{z}}+|z|^{2}
$$

On the one hand, by integration by parts

$$
\begin{aligned}
\left(\Pi \partial_{z} \partial_{\bar{z}} u\right)(z) & =\frac{1}{\pi} e^{-\frac{|z|^{2}}{2}} \int_{\mathbb{C}} e^{\bar{w} z-\frac{|w|^{2}}{2}} \partial_{w} \partial_{\bar{w}} u(w) d L(w) \\
& =\frac{1}{\pi} e^{-\frac{|z|^{2}}{2}} \int_{\mathbb{C}} \partial_{w} \partial_{\bar{w}}\left(e^{\bar{w} z-\frac{|w|^{2}}{2}}\right) u(w) d L(w) \\
& =-\frac{1}{2} \Pi u(z)-\frac{1}{2} z \Pi(\bar{w} u)(z)+\frac{1}{4} \Pi\left(|w|^{2} u\right)(z)
\end{aligned}
$$

thus

On the other hand

$$
\Pi H u(z)=2 \Pi u(z)+2 z \Pi(\bar{w} u)(z) .
$$

$$
\begin{aligned}
\partial_{z} \Pi u(z) & =-\frac{1}{\pi} \frac{\bar{z}}{2} e^{-\frac{|z|^{2}}{2}} \int_{\mathbb{C}} e^{\bar{w} z-\frac{|w|^{2}}{2}} u(w) d L(w)+\frac{1}{\pi} e^{-\frac{|z|^{2}}{2}} \int_{\mathbb{C}} e^{\bar{w} z-\frac{|w|^{2}}{2}} \bar{w} u(w) d L(w) \\
& =-\frac{\bar{z}}{2} \Pi u(z)+\Pi(\bar{z} u)(z)
\end{aligned}
$$


then

$$
\partial_{\bar{z}} \partial_{z} \Pi u(z)=-\frac{1}{2} \Pi u(z)+\frac{1}{4}|z|^{2} \Pi u(z)-\frac{z}{2} \Pi(\bar{w} u)(z),
$$

and we get that

$$
H \Pi u(z)=2 \Pi u(z)+2 z \Pi(\bar{w} u)(z)=\Pi H u(z),
$$

which was the claim.

We recall a short version of [ST21, Lemma A.2]:

Lemma C.2. - For all $j, k \geqslant 0$ there exists $C>0$ such that for all $1 \leqslant p \leqslant \infty$ and $v \in \mathcal{E}$,

$$
\left\|\partial_{\bar{z}}^{j} \partial_{z}^{k}\left(|v|^{2}\right)\right\|_{L^{p}(\mathbb{C})} \leqslant C\|v\|_{L^{2 p}(\mathbb{C})}^{2} .
$$

\section{BIBLIOGRAPHY}

[ABN06] Amandine Aftalion, Xavier Blanc, and Francis Nier, Lowest Landau level functional and Bargmann spaces for Bose-Einstein condensates, J. Funct. Anal. 241 (2006), no. 2, 661-702. $\uparrow 1599$

[ANS19] Jack Arbunich, Irina Nenciu, and Christof Sparber, Stability and instability properties of rotating Bose-Einstein condensates, Lett. Math. Phys. 109 (2019), no. 6, 1415-1432. $\uparrow 1598$

[Bam17] Dario Bambusi, Reducibility of 1-d Schrödinger equation with time quasiperiodic unbounded perturbations. II, Commun. Math. Phys. 353 (2017), no. 1, 353-378. ^1614

[Bam18]__ Reducibility of 1-d Schrödinger equation with time quasiperiodic unbounded perturbations. I, Trans. Am. Math. Soc. 370 (2018), no. 3, 1823-1865. ^1614

[BBCE17] Anxo Biasi, Piotr Bizoń, Ben Craps, and Oleg Evnin, Exact lowest-Landau-level solutions for vortex precession in Bose-Einstein condensates, Phys. Rev. A 96 (2017), no. 5, article no. 053615. $\uparrow 1599$

[BBE19] Anxo Biasi, Piotr Bizoń, and Oleg Evnin, Solvable cubic resonant systems, Commun. Math. Phys. 369 (2019), no. 2, 433-456. ^1599

[BGMR18] Dario Bambusi, Benoît Grébert, Alberto Maspero, and Didier Robert, Reducibility of the quantum harmonic oscillator in d-dimensions with polynomial time-dependent perturbation, Anal. PDE 11 (2018), no. 3, 775-799. ^1598, 1601, 1611, 1614

[BGMR21] _ Growth of Sobolev norms for abstract linear Schrödinger equation, J. Eur. Math. Soc. (JEMS) 23 (2021), no. 2, 557-583. $\uparrow 1598$

[BM18] Dario Bambusi and Riccardo Montalto, Reducibility of 1-d Schrödinger equation with time quasiperiodic unbounded perturbations. III, J. Math. Phys. 59 (2018), no. 12, article no. 122702. $\uparrow 1614$

[Bou99a] Jean Bourgain, Growth of Sobolev norms in linear Schrödinger equations with quasiperiodic potential, Commun. Math. Phys. 204 (1999), no. 1, 207-247. 1598

[Bou99b] _ On growth of Sobolev norms in linear Schrödinger equations with smooth time-dependent potential, J. Anal. Math. 77 (1999), 315-348. ^1598, 1602

[Car91] Eric A. Carlen, Some integral identities and inequalities for entire functions and their application to the coherent state transform, J. Funct. Anal. 97 (1991), no. 1, 231-249. $\uparrow 1603$ 
[Del10] Jean-Marc Delort, Growth of Sobolev norms of solutions of linear Schrödinger equations on some compact manifolds, Int. Math. Res. Not. 2010 (2010), no. 12, 2305-2328. 11598 , 1601, 1602

[Del14]_ Growth of Sobolev norms for solutions of time dependent Schrödinger operators with harmonic oscillator potential, Commun. Partial Differ. Equations 39 (2014), no. 1, 1-33. $\uparrow 1597,1598$

[EK09] Hakan L. Eliasson and Sergei B. Kuksin, On reducibility of Schrödinger equations with quasiperiodic in time potentials, Commun. Math. Phys. 286 (2009), no. 1, 125-135. $\uparrow 1614$

[Eli01] Hakan L. Eliasson, Almost reducibility of linear quasi-periodic systems, Smooth ergodic theory and its applications (Seattle, WA, 1999), Proceedings of Symposia in Pure Mathematics, vol. 69, American Mathematical Society, 2001, pp. 679-705. 11614

[FGH16] Erwan Faou, Pierre Germain, and Zaher Hani, The weakly nonlinear large box limit of the $2 D$ cubic NLS, J. Am. Math. Soc. 29 (2016), no. 4, 915-982. 11601

[FR20] Erwan Faou and Pierre Raphaël, On weakly turbulent solutions to the perturbed linear harmonic oscillator, https://arxiv.org/abs/2006.08206, 2020. $\uparrow 1599$

[FZ12] Daoyuan Fang and Qidi Zhang, On growth of Sobolev norms in linear Schrödinger equations with time-dependent Gevrey potential, J. Dyn. Differ. Equations 24 (2012), no. 2, 151-180. $\uparrow 1598$

[GGT19] Patrick Gérard, Pierre Germain, and Laurent Thomann, On the cubic lowest Landau level equation, Arch. Ration. Mech. Anal. 231 (2019), no. 2, 1073-1128. ^1598, 1599, 1602, 1603, 1604, 1607, 1614

[GHT16] Pierre Germain, Zaher Hani, and Laurent Thomann, On the continuous resonant equation for NLS. I. Deterministic analysis, J. Math. Pures Appl. 105 (2016), no. 1, 131-163. $\uparrow 1599,1601$

[GIP09] Benoît Grébert, Rafik Imekraz, and Éric Paturel, Normal forms for semilinear quantum harmonic oscillators, Commun. Math. Phys. 291 (2009), no. 3, 763-798. 11614

[GP19] Benoît Grébert and Éric Paturel, On reducibility of quantum harmonic oscillator on $\mathbb{R}^{d}$ with quasiperiodic in time potential, Ann. Fac. Sci. Toulouse, Math. 28 (2019), no. 5, 977-1014. $\uparrow 1614$

[GPT13] Benoît Grébert, Éric Paturel, and Laurent Thomann, Beating effects in cubic Schrödinger systems and growth of Sobolev norms, Nonlinearity 26 (2013), no. 5, 13611376. $\uparrow 1598$

[GT11] Benoît Grébert and Laurent Thomann, Kam for the quantum harmonic oscillator, Commun. Math. Phys. 307 (2011), no. 2, 383-427. ^1599, 1613, 1614, 1615

[Hel84] Bernard Helffer, Théorie spectrale pour des opérateurs globalement elliptiques, Astérisque, vol. 112, Société Mathématique de France, 1984. 1609

[HM20] Emanuele Haus and Alberto Maspero, Growth of Sobolev norms in time dependent semiclassical anharmonic oscillators, J. Funct. Anal. 278 (2020), no. 2, article no. 108316. $\uparrow 1598$

[LW19] Zhenguo Liang and Zhiguo Wang, Reducibility of quantum harmonic oscillator on $\mathbb{R}^{d}$ with differential and quasi-periodic in time potential, J. Differ. Equations 267 (2019), no. 5, 3355-3395. 11614

[LZZ21] Zhenguo Liang, Zhiyan Zhao, and Qi Zhou, 1-d quantum harmonic oscillator with time quasi-periodic quadratic perturbation: reducibility and growth of Sobolev norms, J. Math. Pures Appl. 146 (2021), 158-182. $\uparrow 1598$

[Mas19] Alberto Maspero, Lower bounds on the growth of Sobolev norms in some linear time dependent Schrödinger equations, Math. Res. Lett. 26 (2019), no. 4, 1197-1215. ^1598 
[MR17] Alberto Maspero and Didier Robert, On time dependent Schrödinger equations: global well-posedness and growth of Sobolev norms, J. Funct. Anal. 273 (2017), no. 2, 721-781. $\uparrow 1596,1597,1598,1610,1611$

[Nie07] Francis Nier, Bose-Einstein condensates in the lowest Landau level: Hamiltonian dynamics, Rev. Math. Phys. 19 (2007), no. 1, 101-130. $\uparrow 1599$

[Par10] Alberto Parmeggiani, Spectral theory of non-commutative harmonic oscillators: an introduction, Lecture Notes in Mathematics, vol. 1992, Springer, 2010. $\uparrow 1609$

[Rob87] Didier Robert, Autour de l'approximation semi-classique, Progress in Mathematics, vol. 68, Birkhäuser, 1987. $\uparrow 1609$

[ST21] Valentin Schwinte and Laurent Thomann, Growth of Sobolev norms for coupled Lowest Landau Level equations, Pure Appl. Anal. 3 (2021), no. 1, 189-222. $\uparrow 1599,1601,1603$, $1604,1605,1616$

[Wan08a] Wei-Min Wang, Logarithmic bounds on Sobolev norms for time-dependant linear Schrödinger equations, Commun. Partial Differ. Equations 33 (2008), no. 12, 21642179. $\uparrow 1598$

[Wan08b] _ Pure point spectrum of the Floquet Hamiltonian for the quantum harmonic oscillator under time quasi-periodic perturbations, Commun. Math. Phys. 277 (2008), no. $2,459-496 . \uparrow 1614$

[YZ04] Kenji Yajima and Guoping Zhang, Local smoothing property and Strichartz inequality for Schrödinger equations with potentials superquadratic at infinity, J. Differ. Equations 202 (2004), no. 1, 81-110. $\uparrow 1611$

[Zhu12] Kehe Zhu, Analysis on Fock spaces, Graduate Texts in Mathematics, vol. 263, Springer, 2012. $\uparrow 1602$

Manuscript received on 2nd June 2020, revised on 25th February 2021, accepted on 14th April 2021.

Recommended by Editor F. Hérau.

Published under license CC BY 4.0.

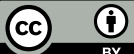

This journal is a member of Centre Mersenne.

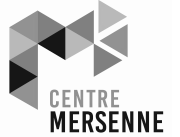

Laurent THOMANN

Institut Élie Cartan,

Université de Lorraine,

B.P. 70239, F-54506

Vandœuvre-lès-Nancy Cedex, (France)

laurent.thomann@univ-lorraine.fr 